\title{
OTIMIZAÇÃO DE PROCESSOS INDUSTRIAIS: CONTROLE AVANÇADO NÃO É A ÚNICA SAÍDA*
}

Rafael Henrique Alves Gomes ${ }^{1}$ Freddy Teixeira Pelucio

\section{Resumo}

A automação em processos industriais agrega qualidade, padronização e segurança aos produtos e pessoas envolvidas. Entretanto, as demandas de mercado, custos agregados e a concorrência exigem melhorias que podem ser alcançadas através de métodos de otimização. Este artigo apresenta algumas metodologias e ferramentas para otimização de processos industriais que visam desvincular a obrigatoriedade de que Técnicas de Controle Avançado em métodos de otimização são a única solução capaz de oferecer resultados satisfatórios para aumento de produtividade. Os recursos expostos abordam a gestão de malhas de controle, gestão de alarmes e o cálculo do indicador global de eficiência, o OEE.

Palavras-chave: Automação industrial; Controle avançado; Gestão; Otimização.

\section{INDUSTRIAL PROCESS OPTIMIZATION: ADVANCED CONTROL IS NOT THE ONLY WAY}

\section{Abstract}

The automation in industrial processes adds quality, standardization and safety of products and people involved. However, industrial automation requires improvements that can be achieved through optimization methods. This article presents new methodologies and tools for optimization of industrial processes to untie the obligation of Advanced Control Techniques in optimization methods. Exposed methodologies address the mesh management control, management of alarms and the calculation of Global Efficiency Indicator, OEE.

Keywords: Advanced control; Industrial automation; Management; Optimization.

1 Mestre em Engenharia Elétrica pela Universidade Federal de Minas Gerais, Analista de Automação na APTA Sistemas de Automação Ltda., Belo Horizonte, Minas Gerais, Brasil.

2 Diretor Comercial na APTA Sistemas de Automação Ltda., Belo Horizonte, Minas Gerais, Brasil. 


\section{INTRODUÇÃO}

A evolução tecnológica oferece possibilidades capazes de mudar o cenário produtivo de uma planta industrial para outro mais favorável. A automação industrial é um exemplo típico, capaz de transformar cenários. Processos automatizados entregam resultados confiáveis, padronizados e com segurança operacional. Contudo, a exigência de melhoria contínua nos processos e maximização do lucro impulsionam o desenvolvimento de técnicas capazes de gerar aumentos significativos nos resultados. Nesse sentido, surgem os primeiros questionamentos a respeito da possibilidade de como tornar processos ainda mais eficientes. Controle Avançado é uma solução possível.

O Controle Avançado de Processos tem a premissa de maximizar o retorno do investimento, minimizar a utilização de matérias-primas e recursos humanos, através de modelos matemáticos capazes de, automaticamente, se adaptarem a diversas mudanças de operacionais recalculando seus parâmetros [1], ou seja, capazes de influenciar diretamente o comportamento do processo de forma autônoma, afim de manter suas premissas atendidas. Modelos adaptativos, preditivos, neurais e fuzzy, são exemplos básicos para o desenvolvimento de Técnicas de Controle Avançado. Entretanto, sua utilização exige, na maioria das vezes, estudos complexos relacionados à modelagem matemática, construção dos controladores e hardwares específicos. Embora haja potencialidades claras em Técnicas de Controle Avançado, sua implantação pode representar um obstáculo e, consequentemente, ser impeditivo no processo de otimização industrial criando um paradigma entre Otimização de Processos Industrial e Técnicas de Controle Avançado.

Neste trabalho são apresentadas ferramentas, metodologias e técnicas que são capazes de criar um cenário favorável para otimização de processos sem a necessidade de implantação de Técnicas de Controle Avançado, modificações na planta ou processo vigente. A quebra do paradigma entre Otimização de Processos e Controle Avançado é a chave para que diversos processos industriais possam se tornar competitivos e alcançar resultados satisfatórios. São abordados neste trabalho técnicas para Gestão de Malhas de Controle PID, Gestão de Alarmes e o Cálculo do Índice Global de Eficiência (OEE), utilizadas em conjunto ou separadamente, que resultarão em um framework capaz de maximizar os ganhos de um processo e aproximar todas as áreas: operação, manutenção e gerência, tornando-as diretamente responsáveis pelos bons resultados que serão obtidos.

Este trabalho contém cinco seções organizadas da seguinte forma: i) a seção 1 apresenta a introdução do trabalho e aspectos gerais; ii) a seção 2 aborda técnicas de Gestão de Malhas de Controle; iii) a seção 3 aborda técnicas de Gestão de Alarmes; iv) a seção 4 aborda aspectos do OEE; e por fim, a conclusão do trabalho é apresentada na seção 5.

\section{GESTÃO DE MALHAS DE CONTROLE}

Malhas de Controle, em processos industriais, agregam maior segurança de operação, mantém a fabricação previsível, asseguram eficácia e qualidade no processo. Entretanto, para que tais características se mantenham é necessário garantir que todos os equipamentos de medição e calibração que compõem uma malha de controle estejam calibrados corretamente, que os ganhos para parametrização da malha estejam configurados corretamente e que, principalmente, 
sejam utilizadas de maneira automática, de acordo com controladores programáveis e não pela expertise de operadores.

Malhas de controle clássicas, principalmente do tipo PI e PID, representam cerca de $90 \%$ de todas as aplicações de malhas de controle em ambientes industriais [2], principalmente pela eficiência, facilidade de operação e sintonia. Entretanto, em diversas aplicações industriais, quedas de performance podem estar associadas diretamente às mesmas malhas de controles, desde que hajam:

- Malhas PID mal sintonizadas;

- Interferências em malhas saudáveis causadas por outras mal sintonizadas;

- Operação em Modo Manual das Malhas PID.

Intuitivamente, na busca por melhores resultados, técnicas de controle avançado como por exemplo, controladores neurais e fuzzy [3], são considerados opções viáveis. Contudo, sem a garantia de as malhas de controle PI e PID estejam corretamente sintonizadas e operando com a máxima performance possível, o uso de algoritmos especiais e técnicas de controle avançado podem representar um erro.

A adoção de novas soluções de controle não iria solucionar os problemas de queda de performance, mas talvez minimizá-los com soluções mais complexas. Modelos e algoritmos de controle avançado só devem ser utilizados a partir do momento que malhas de controle clássicas não ofereçam os ganhos almejados, desde que operem com máxima eficiência.

O desafio é manter as malhas PI e PID corretamente parametrizadas durante todo 0 processo. Nesse contexto, alguns pontos precisam ser observados, dos quais destacam-se os seguintes questionamentos:

- A aferição e manutenção dos equipamentos de medição e atuação são realizadas com frequência e da maneira correta?;

- A sintonia dos controladores foi realizada de maneira conservadora?;

- A operação das malhas de controle está predominantemente em modo automático?

Boas práticas na administração das malhas de controle é um fator decisivo para o bom desempenho e garantia de resultados. Nesse processo, gerir malhas de controle fornece condições para identificar possíveis pontos de falhas e então, atuar de maneira correta na solução. A adoção de uma metodologia para gestão de malhas é um fator decisivo na otimização de processos industriais.

Diversas aplicações são desenvolvidas para auxiliar na otimização de processos industriais que permitem monitorar o desempenho dos sistemas de controle, identificando problemas, isolando as causas e fazendo recomendações de correção. Existem plataforma WEB capazes de avaliar a eficiência de malhas de controle através de diversas métricas de qualidade e desempenho, como por exemplo a variância na saída de controle, o erro médio absoluto calculado entre o set-point e a variável medida e o tempo em que a malha permaneceu em modo automático. Com tais informações, a plataforma WEB fornece KPIs (Key Performance Indicator) que quantificam a eficiência do controle em tempo real. Além de disponibilizarem relatórios de desempenhos e ranking das piores malhas. Ferramentas para gestão de malhas atuam principalmente como auditores que apontarão erros e pontos de melhoria, pois se malhas de controle não são aferidas, certamente elas não estarão funcionando com máxima eficiência.

Sintonizar malhas PID é um processo que exige experiência e deve ser amparado por procedimentos matemáticos consolidados. A sintonia empírica de controladores PID pode diminuir o desempenho de um processo consideravelmente. Dessa forma, assim 
como para o gerenciamento de malhas de controle, existem ferramentas consolidadas para calcular, de forma segura, os valores ideais dos ganhos de um controlador PI ou PID, por exemplo. O uso associado de ferramentas para gestão de malhas de controle e sintonia de controladores cria a possibilidade de se atingir um cenário onde a performance de um processo industrial pode ser maximizada, apenas com pequenos investimentos e uma séria mudança de postura do que se refere a encarar malhas de controle como ativos em um processo industrial.

\section{GESTÃO DE ALARMES}

A definição formal do que é um alarme pode ser encontrada em [4] que o trata como um anúncio ao operador iniciado por uma condição de mau funcionamento de um equipamento, desvio de processo ou condição anormal que requer uma ação. Nesse sentido, a lógica apontaria para um cenário no qual o operador, ao notar um alarme, tomaria uma decisão afim de investigar e corrigir sua origem. Contudo, na maioria das vezes os alarmes não agregam nenhuma informação ao processo e são menosprezados. Desta maneira, uma ferramenta que, teoricamente, seria útil para o processo torna-se algo inútil que somente o sobrecarrega com dados sem significado. A perda de credibilidade dos alarmes em processos industriais ocorreu na mesma velocidade do crescimento tecnológico. Antes do advento dos Sistemas de Controle Distribuídos (DCS) e SCADA, os alarmes eram normalmente dispositivos visuais e/ou sonoros montados em painéis que estavam sempre visíveis e disponíveis à operação. Os sistemas de automação modernos eliminaram os custos de criação de alarmes e, consequentemente essa facilidade, aliada à falta de metodologia e padrão, fizeram com que essa ferramenta se tornasse parte ineficaz do processo.

Um erro comum na maioria dos sistemas de alarme é associa-los a eventos de operação e registrá-los com a mesma importância. Os eventos são classificados como uma situação que ocorre no processo e tem importância para o operador a título de informação, mas não requer nenhuma ação corretiva; por outro lado, os alarmes são definidos como uma situação que ocorre no processo e exige que o operador tome providências em relação a ela sob pena de levar o processo a situações de perigo.

Sistemas de alarmes mal projetados e que não auxiliam na melhoria do processo possuem as características comuns que podem ser listadas a seguir:

- Os operadores ignoram, com frequência, os alarmes;

- Não existe prioridade entre alarmes;

- Operadores tem liberdade suprimir e alterar configurações de alarmes;

- Na ocorrência de alarmes, os operadores não sabem qual atitude tomar.

Aliar o excesso de informação sem credibilidade e significado à dependência da resposta humana para interpretação dos alarmes é o suficiente para que sistemas de gerenciamento de alarmes sejam fundamentais em um processo industrial que vise melhoria contínua. Existem dois padrões internacionais que tratam as premissas que sistemas de gerenciamento de alarmes devem atender, sendo eles:

- EEMUA [5] - Sistemas de Alarmes: Um Guia de Projetos, Gerenciamento e Compra

- ISA 18.2 [4] - Sistemas de Gerenciamento de Alarmes Industriais.

Segundo a regulamentação da [4], um bom sistema para a gestão de alarmes deve contribuir para que o cenário de alarmes atinja os seguintes indicadores:

- Quantidade máxima de alarmes que podem estar ativos, por hora, considerando um operador é de seis alarmes; 
- Porcentagem com mais de 30 alarmes ativos, em uma hora, deve sempre ser menor que $1 \%$;

- Porcentagem da quantidade de alarmes "bad actors" (alarmes que são causa de outros alarmes menores) deve sempre ser menor que $5 \%$ da quantidade total de alarmes;

- Número de alarmes suprimidos ou alterados, durante todo o processo, sem permissão deve ser zero;

- Alarmes com Prioridade Baixa devem ser $80 \%$ do total de alarmes, enquanto os de prioridade média são $15 \%$; somente $5 \%$ devem ser alarmes com prioridade alta.

Um sistema de gerenciamento de alarmes, baseados nas premissas do padrão EEMUA, deve coletar as informações dos alarmes e analisa-las continuamente. Somente a coleta de informações do chão de fábrica já pode apresentar um desafio. Boas soluções em gerenciamento de alarmes são aquelas que conseguem trazer as informações de campo e formata-las de maneira clara e confiável para a sala de controle. Casos de mau gerenciamento de alarmes induziram erros de operação que foram responsáveis por tragédias como as ocorridas na Usina Nuclear de Chernobyl, em 1986 e a explosão da plataforma BP Oil, em 2010.

Certas funcionalidades tornam algumas ferramentas de gerenciamento de alarmes capazes de interagir com o processo gerando informações seguras e confiáveis. Soluções em gerenciamento de alarmes devem possuir, segundo [6], conectividade, processamento computacional isolado do sistema de controle, fácil acesso às interfaces, alta capacidade de armazenamento para histórico e fácil manutenção. Um sistema de gestão de alarmes que seja capaz de reunir todas essas características é capaz de agregar bons resultados ao processo. Um alarme, para ser considerado confiável e útil deve:

- Ser relevante e único, ou seja, que a informação trazida pelo alarme seja suficiente para que uma ação preventiva ou corretiva seja tomada;

- Possua prioridade e seja definido no tempo;

- Inteligível e suficiente para orientar uma ação do operador.

O gerenciamento de alarmes é um ciclo de melhoria contínua, ou seja, dinâmico e requer atenção para que bons resultados sejam alcançados. O gerenciamento de alarmes é composto em sete fases, definidas na ISA 18.2. As ferramentas de gestão de alarmes oferecem facilidades que não alcançaríamos sem elas, como por exemplo, identificação de alarmes com maior incidência, áreas com maior número de alarmes, produção de relatórios de indicadores de desempenho. Contudo, a utilização isolada das ferramentas não trará os resultados esperados com a gestão dos alarmes. A figura 1 destaca os passos para que gerenciamento de alarmes aconteça de maneira eficaz e a maneira contínua em que se relacionam. 


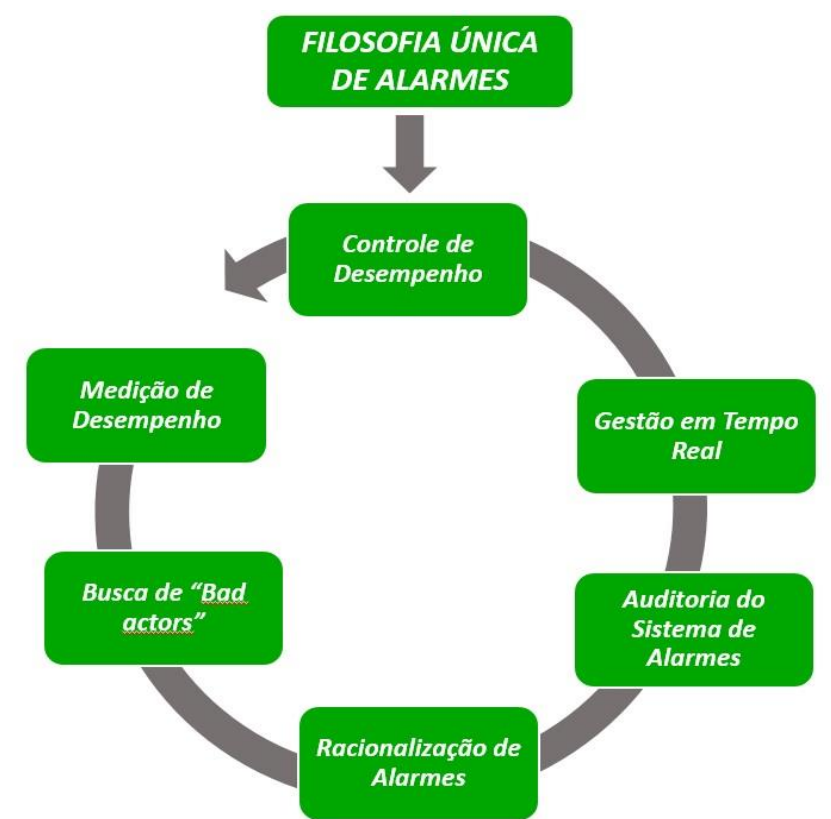

Figura 1 - Ciclo para gerenciamento de alarmes.

No início da implementação de um sistema de gerenciamento de alarmes é importante não atacar o universo de todos os alarmes de uma só vez. É interessante que sejam selecionadas algumas áreas e, de maneira gradativa, a nova filosofia de gerenciamento seja inserida. Com isso, será possível obter resultados de maneira mais simples e que comprovem sua utilidade.

Sistemas de gerenciamento de alarmes oferecem maior estabilidade operacional. Com os relatórios e KPIs fornecidos pelos sistemas é possível identificar pontos de falhas, gargalos na operação e agir de maneira pontual. Embora os alarmes sejam mensagens em tempo real, sistemas de gerenciamento são capazes de gerar históricos e com isso construir relatórios e índices de desempenho.

O emprego de ferramentas de gestão de alarmes oferece benefícios ao processo produtivo que irão refletir no aumento da produtividade através do aumento da estabilidade operacional. O OEE (Índice de Eficiência Global) é influenciado diretamente com os bons resultados trazidos pela gestão dos alarmes, pois os fatores de eficiência, qualidade e performance são afetados diretamente quando os alarmes oferecem informações confiáveis e seguras para atuação. Planos efetivos de manutenção e melhoria contínua podem ser elaborados a partir de falhas identificadas pelos sistemas de gerenciamento, e ainda diminuir a interferência operacional, sem metodologia, na resolução dos problemas.

\section{OEE - ÍNDICE GLOBAL DE EFICIÊNCIA}

Indicadores de eficiência são, muitas vezes, calculados a partir de planilhas alimentadas com dados manuais e que, na maioria dos casos, são apontados em períodos posteriores às grandes paradas no processo. Com isso, quando calculadas, as métricas de eficiências podem representar cenários contrários a realidade do processo ou até mesmo mascará-los. Atualmente, existem maneiras de coletar informações em tempo real e em conjunto com algoritmos capazes de calcular a eficiência de um equipamento de forma transparente e livre de erros. Para isso, é necessário transformar dados em informação. A grande diferença entre eles é que a informação fornece condições de prever e planejar. 
Um erro comum na avaliação da eficiência de um processo é considera-la uma razão entre os insumos necessários e o produto final adquirido, ou seja, manter os olhos na eficiência somente pela ótica da produção. Isso pode representar armadilha. O OEE não é um índice utilizado somente para PCP (Planejamento e Controle da Produção), mas deve ser utilizado também pelo PCM (Planejamento e Controle da Manutenção) como chave para avaliar problemas e gargalos no processo. Para evitá-la é necessário avaliar, inicialmente, algumas questões:

- Os equipamentos do processo param constantemente?

- Os motivos de todas as paradas são conhecidos? São paradas por defeito ou condições de processo?

- As pequenas paradas são consideradas no somatório do tempo em que o equipamento esteve parado?

- Os dados referentes a paradas e defeitos, quando existem, são coletados automaticamente ou dependem da ação de operadores?

- Qual o tempo gasto para reestabelecer equipamentos com defeitos ou falhas?

- Existem planos de manutenção coerentes e que são realizados com seriedade?

- Qual o impacto na produção devido a problemas com equipamentos isoladamente?

$\mathrm{Na}$ década de 70, a necessidade em manter os equipamentos sempre funcionais levou ao desenvolvimento de uma metodologia de manutenção denominada TPM (Total Productive Manufacturing) [7], que maximizava a eficiência de um equipamento através de um plano otimizado de manutenção que envolvia desde o operador até os gerentes de processo [8]. O objetivo da TPM é eliminar ou ao menos diminuir as perdas por manutenção, aumentar a eficiência e o tempo útil das máquinas, resultando no aumento global da produtividade industrial. Contudo, ainda era necessário mensurar se a metodologia TPM era eficaz em relação à sua proposta. Nesse sentido, foi criado o índice OEE (Overall Equipment Effectiveness). O OEE é um índice calculado em tempo real a partir de métricas de eficiência do equipamento. Ele fornece um valor que pode ser utilizado como métrica global de eficiência capaz de proporcionar ganhos de qualidade e produtividade, permitindo atuar nas perdas de produção e diminuindo o investimento em novos equipamentos.

$\mathrm{O}$ uso do OEE cria métricas padronizadas que auxiliam equipes de manutenção a aumentarem a performance de cada equipamento e, posteriormente reduzir a perda que aquele equipamento causa no processo.

Existem diversas variações para o cálculo de OEE, porém a metodologia mais consistente utiliza apenas três métricas básicas, sendo: a disponibilidade, a performance e a taxa de qualidade do equipamento [8].

A principal variável para o cálculo do OEE é o tempo em que o equipamento permaneceu operando ou parado. É preciso gerar intervalos nos quais se compara o tempo no qual a máquina agrega valor ao produto e soma total das perdas de tempo enquanto a mesma estiver parada [9]. Para o cálculo do OEE é necessário, inicialmente, definir quais os motivos de parada dos equipamentos. Os seis principais motivos de perda de tempo em um processo produtivo estão relacionados a parada de equipamentos e podem ser observados na Figura 2. 


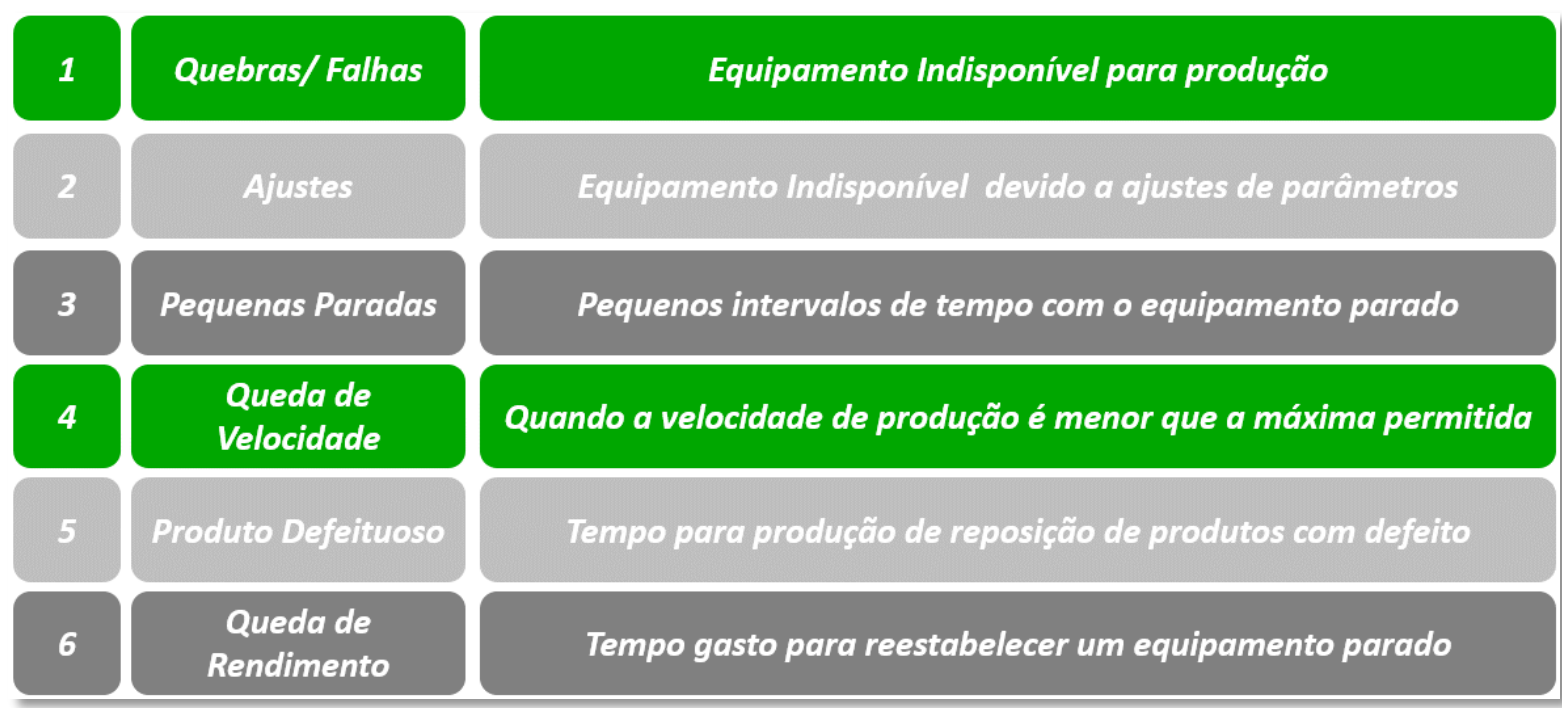

Figura 2 - Interface gráfica para sintonia de malhas de controle

O tempo no qual o equipamento ou grupo de equipamentos permaneceu parado, por qualquer motivo, deve ser contabilizado e de maneira automática. Apontamentos incorretos sobre o tempo de paradas e o motivo do mesmo podem mascarar resultados e comprometer todo o processo. Assim, os dados de processos precisam ser coletados em fontes confiáveis e persistidos em bancos de dados seguros e de rápido acesso.

O segundo passo é calcular as métricas de qualidade, disponibilidade e performance para cada equipamento. As métricas responsáveis pelo cálculo do OEE estão ligadas diretamente com os seis motivos de paradas, definidos na Figura 3.

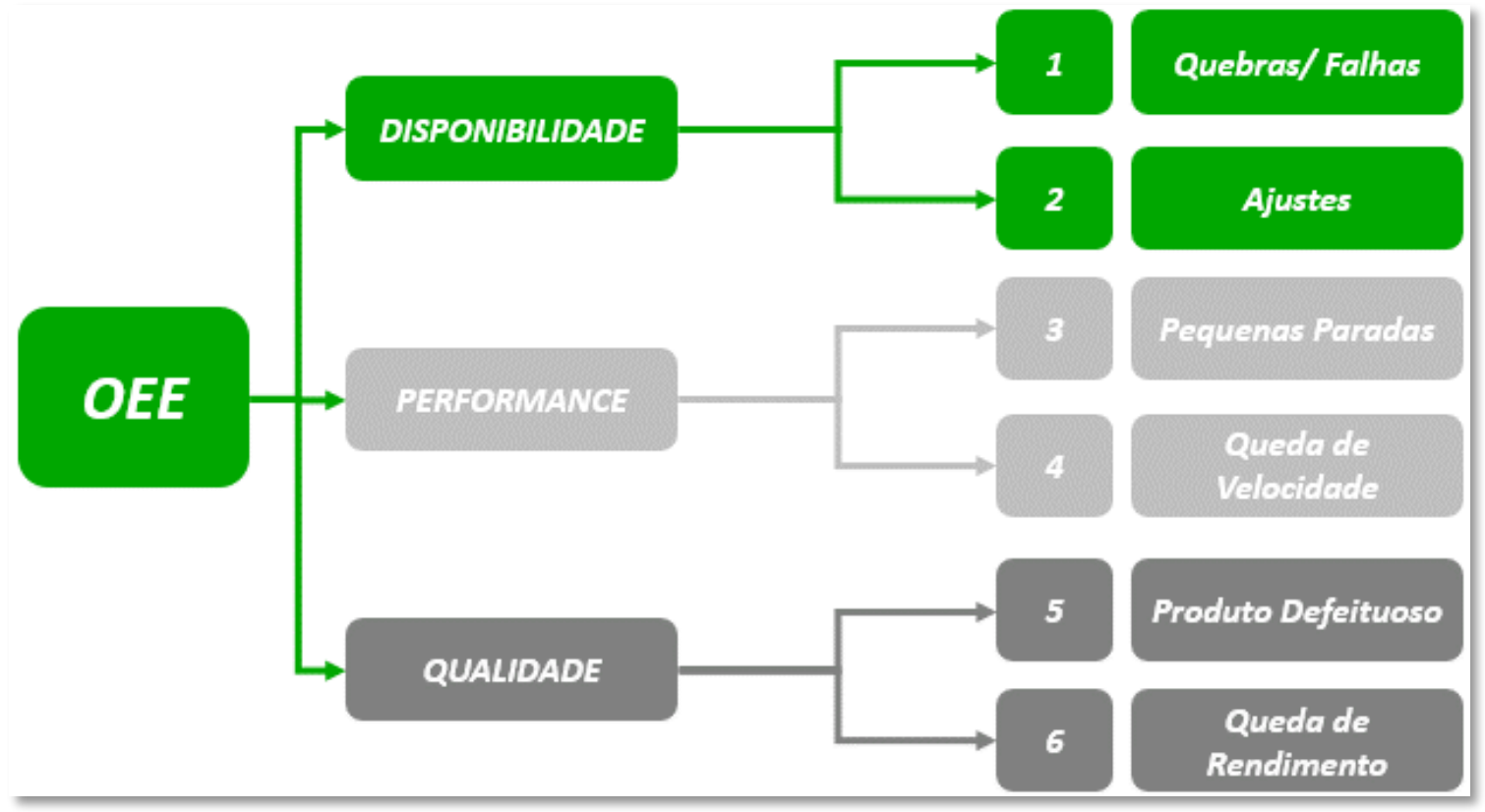

Figura 3 - Interface gráfica para sintonia de malhas de controle

Por fim, o valor do OEE é obtido pelo produto das três métricas, disponibilidade, performance e qualidade. Em [10], o OEE é definido na equação 1:

$$
O E E=D * P * Q
$$


Em [10], as métricas D, P e Q são calculados de acordo com as equações 2, 3 e 4.

$$
\begin{array}{r}
D=\frac{\text { tempo total do processo-tempo parado }}{\text { tempo total do processo }} \\
P=\frac{\text { tempo estimado para produção } * \text { qnt produzida nesse tempo estimado }}{\text { tempo total do processo-tempo parado }} \\
Q=\frac{\text { quantidade produzida-quantidade rejeitada }}{\text { quantidade produzida }}
\end{array}
$$

O valor do OEE deve ser calculado constantemente a medida que os dados produção são coletados e validados automaticamente para evitar manobras e falhas no processo. Atingir valores altos de OEE como, por exemplo, 90\% é uma tarefa muito difícil. Valores de benchmark mundiais para OEE alcançam valores próximos a $85 \%$, o que reflete $90 \%$ de disponibilidade, $95 \%$ de performance e $99 \%$ de qualidade. 0 OEE como métrica é uma direção que indicará um sentido de melhoria. Ele desmembra a eficiência de um equipamento nas três principais métricas que podem ser corrigidas separadamente. A figura 4 destaca o impacto da utilização do OEE sobre a disponibilidade, performance e qualidade do processo, aumentando significativamente tais parâmetros.

\begin{tabular}{|r|c|c|c|c|}
\cline { 2 - 5 } \multicolumn{1}{c|}{} & \multicolumn{2}{c|}{$\begin{array}{c}\text { Compahia Produção de Cameras de } \\
\text { Alta Resolução [11] }\end{array}$} & \multicolumn{2}{c|}{ Siderúrgica - Fundição [12] } \\
\cline { 2 - 5 } & Antes & Depois & Antes & Depois \\
\hline Disponibilidade (\%) & 81,5 & 90,2 & 30 & 78 \\
\hline Performance (\%) & 82,5 & 86 & 79 & 50 \\
\hline Qualidade (\%) & 89,8 & 96,1 & 99 & 98 \\
\hline OEE (\%) & $\mathbf{6 0 , 4}$ & $\mathbf{7 4 , 5}$ & $\mathbf{2 3 , 4}$ & 38,4 \\
\hline
\end{tabular}

Figura 4 - Impacto do OEE sobre a produtividade

A utilização do OEE deve sempre vir acompanhada de ações, uma vez que ele altera a ótica na qual a eficiência do processo é observada. Todo equipamento é importante e as perdas estão diretamente ligadas ao tempo em que cada equipamento permanece parado. O OEE é um fator para tomada de decisões estratégicas. Ele pode ser encarado como uma bússola que aponta para a direção do aumento da eficiência produtiva.

\section{CONCLUSÃO}

O uso de técnicas de controle avançado em processos industriais não deve ser a primeira opção para otimização. Nesse artigo foram apresentadas diversas soluções que podem ser utilizadas em conjunto ou separadamente que oferecerão ganhos significativos em relação a disponibilidade, qualidade de produção e lucratividade. Todas as soluções apresentadas podem ser implantadas sem intervenções físicas em processos e representam investimento pequenos, frente aos ganhos possíveis. Em 
épocas de crise econômica e a crescente necessidade em ser competitivo obriga setores de engenharia a serem criativos e responsáveis por soluções que representarão uma saída viável para otimização. Controle Avançado não é a única saída.

\section{REFERÊNCIAS}

1 Gough B, Wilson B, Matovich D. Advanced Model-Based Control for Continuous Process Industries. Dynamic Modeling Control Application for Industries Workshop. 1998; 33-39.

2 Astrom KJ, Hagglund T. The Future of PID Control. Control Enginneering Pratice, 2001.

3 Gomes RHA, Souza FO, Mozelli, LA. Síntese de Controladores Takagi-Sugeno com Taxa de Convergência Pré-Especificada para Sistema com Retardo. XX CBA. 2015

4 ISA A. Management of Alarm System for the Process Industries. 2009

5 EMMUA. Alarm Systems - A Guide to Design Management and Procurement. 2007

6 Cook J. Alarm Management System: The Benefits of Third Party Software. 2014

7 Nakagima, S. Introduction to TPM - Total Productive Maintenance. Productive Press. 1998

8 Bon AT. Implementation of Total Productive Maintenance (TPM) in Automotive Industry. IEEE Symposium on Business, Engeneering and Industrial Applications (ISBEIA). 2011

9 Dornelles JV, Sellito MA. Eficácia Global de Equipamentos (OEE) para Diagnóstico e Melhoria de Produtividade na Indústria de Fundição. GEINTECH. 2015

10 Batumalay K, Santhapparaj AS. Overall Equipment Effectiveness (OEE) Through Total Productive Maintenance (TPM), Practices - A Study across the Malaysian Industries. 2008

11 Zhu X. Analysis and Improvement of Enterprise's Equipment Effectiveness Based on OEE,IEEE, 2011

12 Dorneless JV, Sellito M A. Eficácia Global de Equipamentos (OEE) para Diagnóstico e Melhoria de Produtividade na Indústria de Fundição. GEINTECH, 2015 